\section{Review}

Correspondence

Michael Givskov

immg@pop.dtu.dk

\title{
Quorum sensing inhibitors: a bargain of effects
}

\author{
Thomas B. Rasmussen and Michael Givskov
Centre for Biomedical Microbiology, BioCentrum-DTU, Technical University of Denmark, DK-2800 Kgs Lyngby, Denmark

\begin{abstract}
Many opportunistic pathogenic bacteria rely on quorum sensing (OS) circuits as central regulators of virulence expression. In Pseudomonas aeruginosa, QS-regulated gene expression contributes to the formation and maintenance of biofilms and their tolerance to conventional antimicrobials and the host innate immune system. Therefore, QS is an obvious target for a novel class of antimicrobial drugs which would function to efficiently block reception of the cognate QS signals in vivo, and thereby be capable of inducing chemical attenuation of pathogens. As aS is not directly involved in processes essential for growth of the bacteria, inhibition of OS does not impose harsh selective pressure for development of resistance as with antibiotics. Numerous chemical libraries of both natural and synthetic origin have been screened and several QS-inhibitory compounds have been identified. In animal pulmonary infection models, such inhibitors have proven able to significantly improve clearing of the infecting bacteria and reduce mortality. In addition, several enzymes that are able to inactivate the bacterial OS signal molecules have been identified. This inactivation leads to blockage of QS-mediated virulence of plant pathogens in several models.
\end{abstract}

\section{Quorum sensing}

Bacteria are social organisms capable of interacting with each other and their surroundings. Particularly well described is the ability to coordinate gene expression in accordance with population density, a process termed quorum sensing (QS) (Fuqua et al., 1994). In Gram-negative bacteria this is achieved by production and reception of diffusible signal molecules in the form of acylhomoserine lactones (AHLs) (Fig. 1). The signal molecules are produced by an AHL synthase encoded by homologues of the AHL synthase gene luxI, which was first identified in Vibrio fischeri (Engebrecht \& Silverman, 1984). At low population densities, luxI is constitutively expressed at a low, basal level. Hence, the AHLs accumulate in the surroundings. The LuxR family of receptor/response regulator proteins perceives the AHLs. At a certain threshold concentration of AHL, the signal molecule forms a complex with the receptor protein, which becomes activated. The activated receptor-signal complex in turn forms dimers or multimers with other activated LuxR-AHL complexes. These dimers or multimers function as transcriptional regulators controlling expression of QS-regulated target genes. The QS paradigm states that transcription of QS target genes is activated at a certain population density (which is proportional to the AHL concentration) known as the 'quorum size' - the number of bacteria required to activate the QS system (Eberl, 1999; Fuqua et al., 1994; Salmond et al., 1995; Parsek et al., 1999). In Pseudomonas aeruginosa, however, recent research has shown that each individual QS-regulated gene possesses its own specific quorum size. There is not a single population density at which all QS genes are activated; rather, different genes are activated at different population densities (Hentzer et al., 2003; Wagner et al., 2003; Schuster et al., 2003).

P. aeruginosa is the most common Gram-negative bacterium associated with nosocomial infections, especially of immunocompromised patients (Van Delden \& Iglewski, 1998). In addition, patients suffering from the pulmonary disorder cystic fibrosis are particularly predisposed to infections by $P$. aeruginosa. When these infections become chronic, they are accompanied by substantial damage to the lung tissue and despite aggressive antibiotic chemotherapy the outcome is fatal. For these reasons $P$. aeruginosa is an intensively studied bacterium and it has been revealed that it employs two AHLmediated QS systems to control expression of numerous virulence factors including exoproteases, siderophores and exotoxins as well as several secondary metabolites. In addition $P$. aeruginosa is a formidable biofilm former; production of biofilms is now considered an important pathogenicity trait (Davies et al., 1998; Hentzer et al., 2003; Passador et al., 1996; Winson et al., 1995). The two QS systems in $P$. aeruginosa are arranged in a hierarchical manner with the LasI-LasR system at the top (at least in part) positively controlling activity of the RhlI-RhlR system. The las system utilizes $\mathrm{N}$-(3-oxododecanoyl)-L-homoserine lactone (3-oxo-C12 HSL) whereas the rhl system functions by means of $\mathrm{N}$-butanoylhomoserine lactone as signal molecules (Latifi et al., 1996; Pesci \& Iglewski, 1997; Pesci et al., 1997). Interspaced between these two systems is the Pseudomonas quinolone system, which is a QS system utilizing 2-heptyl-3-hydroxy-4-quionolone (PQS) as signal molecule. The LasR protein regulates expression of the 
A<smiles>[R]C(=O)NC1CCOC1=O</smiles>

B<smiles>O=C1C=C2C(=CCOC2O)O1</smiles>

$\mathrm{C}$<smiles>O=C1C=C(Br)/C(=C/Br)O1</smiles><smiles>[2H]CCCCCCCCC(=O)CC(=O)NC1CCCC1=O</smiles><smiles>CCCCCCCCCC(=O)CC(=O)NC1CCCCC1O</smiles><smiles>O=C(CCCCCCCCF)CC(=O)NC1CCCCC1=O</smiles><smiles>O=C(CCCCCCCCCl)CC(=O)Nc1ccccc1O</smiles>

Fig. 1. A, Basic structure of the acylated homoserine lactones commonly used as signal molcecules by many Gram-negative bacteria (Fuqua et al., 1994). B, Patulin, a OSI isolated from the fungus Pe. coprobium (Rasmussen et al., 2005b). C, Furanone compound 30 (Hentzer et al., 2002). D-G, from agonist to antagonist: D, the native signal molecule 3-oxo-C12 $\mathrm{HSL}$ from the las system in P. aeruginosa (Seed et al., 1995); $\mathrm{E}$, an analogue of 3-oxo-C12 AHL acting as agonist of the las system (Smith et al., 2003a); F, AHL analogue acting as a weak antagonist (Smith et al., 2003a); G, AHL analogue with strong antagonistic effect (Smith et al., 2003b).

pqsABCD and $p q s H$ operons, which are responsible for conversion of anthranilate into PQS. However, a lasR mutant is still able to produce PQS, although at a later stage in growth (Diggle et al., 2003). The PQS signal, if supplemented exogenously, is capable of restoring production of elastase encoded by lasB, which is normally LasR dependent (McKnight et al., 2000; Gallagher et al., 2002; Pesci et al., 1999). Due to their central role in expression of multiple virulence traits in $P$. aeruginosa and the fact that they function by means of low-molecular-mass extracellular signal molecules, the QS systems are clearly potential antibacterial drug targets.

\section{Screening for QS inhibitors}

In order to develop biological screens that will assist in the isolation and identification of QS inhibitors (QSI) it has to be realized which properties are preferentially required for an effective and applicable compound. In essence, the ideal QSI is a low-molecular-mass molecule the activity of which causes a significant reduction in the expression of QS-controlled genes. Second, but equally important, the inhibitor exhibits a high degree of specificity for the QS regulator (i.e. the LuxR homologue) without toxic side effects on either the bacteria or an eventual eukaryotic host. For example, if the QSI does not interfere with basal life processes such as general RNA and protein synthesis of the bacteria, and hence growth, it is conceivable that the selective pressure for development of resistance is minimized. In addition, the QSI should be chemically stable and resistant to metabolism and disposal by the higher host organism.
Several strategies for identifying compounds with QSI activity have been employed by different research groups. One of the most favoured is the use of an AHL biosensor (devoid of a luxI homologue) which harbours a LuxR homologue and a corresponding QS-controlled promoter fused to a reporter gene or operon such as $g f p$ from Aequorea victoria, lacZ from Escherichia coli, the lux gene cluster from $V$. fischeri or the violacein-producing gene cluster from Chromobacterium violaceum. When monitor bacteria encounter AHL signal molecules, the QS-controlled promoter induces expression of the reporter. In the presence of a mixture of exogenous AHLs and QSIs, transcription from the AHL-induced promoter is diminished. Consequently the reporter signal is either reduced or abolished. A screen based on this type of system will report on the presence of a QSI compound by disappearance of the reporter signal. The caveat to this procedure is that factors other than QSI compounds can cause a reduction in the signal. If the biosensor bacterium is challenged with a toxic substance (such as an antibiotic) in sublethal concentrations, growth will be retarded. The underlying reduction in overall protein synthesis and, most importantly, reporter synthesis can be misleading, such that the test substance is scored as a 'false positive'. Therefore, both the production of reporter signal and growth must be carefully monitored and the specific activity of the reporter signal should be calculated and used as a measure of the degree of QS-specific inhibition. Even if these precautions are taken, it can be difficult to obtain reliable information regarding the specificity of a QSinterfering compound that shows additional pleiotropic effects. For example, if the presence of a given compound 
causes a threefold reduction in growth rate and a fivefold reduction in the reporter signal it is impossible to discern whether the decrease in reporter signal is caused by the effect on growth, the inhibition of QS or a mixture of the two - the decrease in reporter signal is not necessarily proportional to the decrease in growth rate. To circumvent these problems Rasmussen et al. (2005a) designed another type of screening system termed the QSI selector (QSIS) (Fig. 2). The QSIS system is based on a bacterium (devoid of a luxI homologue) which harbours a QS-controlled killer gene encoding a toxic gene product. When the bacteria encounter AHLs, the killer gene is expressed and consequently growth of the bacteria will be arrested. In contrast, the presence of a non-toxic QSI compound rescues the bacteria since expression of the killer gene is not activated and the bacteria are able to grow. Hence, this screen provides appearance, i.e. growth of the selector bacteria. The assay requires that the selector bacteria are cast in agar supplemented with the cognate AHL signal molecules. Test substances are placed in wells punched in the plate and diffuse into the agar, creating a concentration gradient. Where the concentration of the inhibitor counterbalances the AHL inducer, it creates a rescue ring of growth surrounding the sample application point. This system has proven useful for isolation of both synthetic compounds and extracts of plants and fungi with QSI activities (Rasmussen et al., 2005a, b; Persson et al., 2005).

\section{Examples of QS inhibitors}

QS systems generally offer three points of attack: the signal generator (LuxI homologue), the signal molecule (AHL) and the signal receptor (LuxR homologue).

\section{Blockage of $\mathrm{AHL}$ production}

To date, the least investigated strategy to interfere with QS is blockage of AHL production. Although a few substrate analogues, including holo-ACP, L/D-S-adenosylhomocysteine, sinefungin and butyryl-S-adenosylmethionine (butyryl-SAM), have been found to be able to block AHL production in vitro (Parsek et al., 1999), none of them have been tested on bacteria in vivo. How these analogues of the AHL building blocks, SAM and acyl-ACP, which are also used in central amino acid and fatty acid catabolism, would affect other cellular functions is presently unknown.

\section{Inactivation of signal molecules}

Another strategy is inactivation or complete degradation of the generated signal molecules. This can be achieved by different methods: chemical degradation, enzymic destruction and metabolism of the AHL. A simple way to achieve inactivation of the AHL signal molecules is by increasing the $\mathrm{pH}$ to above 7; this causes lactonolysis - ring opening - of the AHL (Yates et al., 2002). A number of higher organisms employ this strategy in defence against invading QS bacteria. Plants that are infected with the tissue-macerating plant pathogen Erwinia carotovora will as a first response at the site of attack actively increase pH (Byers et al., 2002). This alkalinization will in turn prevent expression of QScontrolled genes and virulence factors. Several factors influence the kinetics of ring opening. A temperature increase accelerates ring opening but this effect is counteracted by the length of the side chain, which decreases the rate of lactonolysis. These characteristics suggest that in order to be active under physiological conditions, an AHL signal molecule must possess a side chain length of at least four carbons (Byers et al., 2002; Yates et al., 2002). To date, no bacteria producing AHLs with side chains shorter than four carbon atoms have been identified.

Lactonolysis of AHLs can also be accomplished by enzymic activity. Members of the genus Bacillus, including B. cereus, B. mycoides and B. thuringiensis, produce an enzyme, AiiA, specific for degradation of AHLs (Dong et al., 2000, 2001; Wang et al., 2004; Lee et al., 2002). The activity of these enzymes lowers the amount of bioactive AHL signal molecules by catalysing the ring-opening reaction. Within

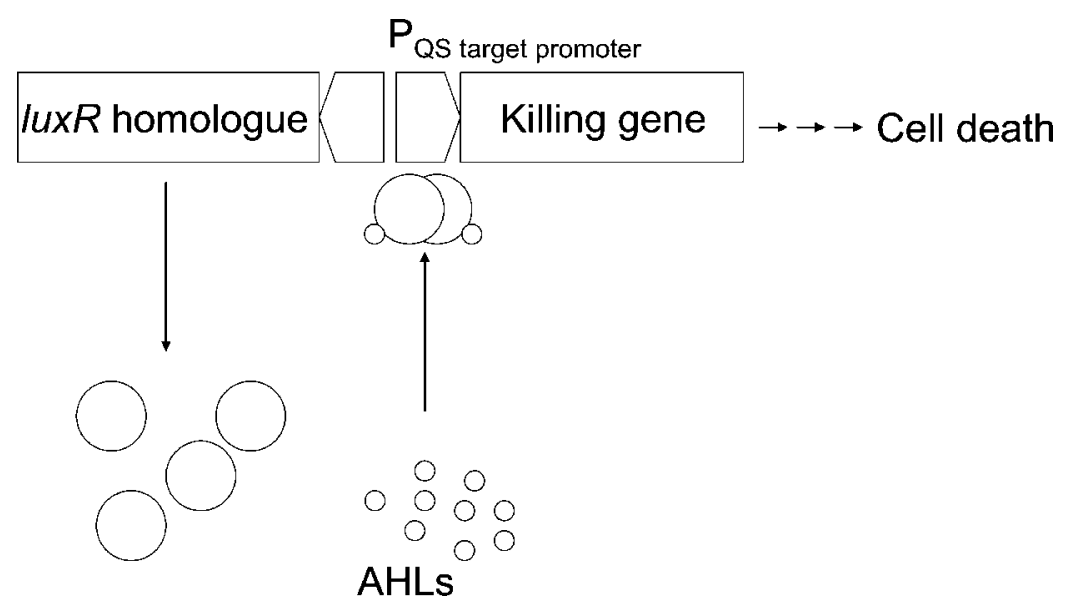

Fig. 2. The OSIS principle. The QSIS bacterium harbours a plasmid carrying a construct consisting of a luxR homologue gene (AHL receptor/response regulator) and a killing function controlled by a OS-regulated promoter. When AHL signal molecules are added exogenously, they form an activated complex with the LuxR homologues, which in turn induces expression of the killing gene, leading to growth arrest of the QSIS bacterium. Conversely, if a OSI is added along with the AHLs, formation of the activated LuxR$\mathrm{AHL}$ complexes or binding of the complexes to the target promoter is prevented. Thus, the killing gene is not expressed and the QSIS bacterium is allowed to grow. Hence, growth of the OSIS bacterium indicates a positive hit presence of a QSI (Rasmussen et al., 2005a). 
$2 \mathrm{~h}$, up to $20 \mu \mathrm{M}$ 3-oxo-C6 HSL can be completely inactivated by a suspension culture producing the enzyme. When Er. carotovora is transformed with a plasmid carrying the aiiA gene, its virulence against potatoes and eggplants is attenuated. In addition, when the plant-colonizing bacterium Pseudomonas fluorescens was transformed with the aiiA gene it was able to prevent soft rot in potatoes caused by $\mathrm{Er}$. carotovora and crown gall disease in tomatoes caused by Agrobacterium tumefaciens. Furthermore, expression of aiiA in transgenic tobacco plants made them much less vulnerable to infection by Er. carotovora compared to their wildtype counterparts (Dong et al., 2001, 2004; Molina et al., 2003). This indeed indicates that enzymic degradation of AHLs would be useful as a means of biocontrol. Production of AHL lactonases is not limited to Bacillus species. Several bacteria including $P$. aeruginosa PAI-A, Arthrobacter sp., Klebsiella pneumoniae, Ag. tumefaciens and Rhodococcus sp., have been found to produce AiiA homologues (Uroz et al., 2003; Carlier et al., 2003; Park et al., 2003; Huang et al., 2003). Other bacteria such as Comamonas sp. have been found to degrade AHLs (Uroz et al., 2003). It seems likely that production of AHL-degrading enzymes constitutes a non-antibiotic-based strategy employed by some bacteria in the competition with AHL producers. Certainly, this class of enzymes has obvious commercial applications, especially in the food manufacturing sector. Unfortunately there is a drawback to the lactonolysis reaction - it is reversible at acidic $\mathrm{pH}$. A ring-opened AHL molecule spontaneously undergoes ring formation if the environment is not alkaline, regardless of the method by which it was opened (chemical or enzymic) (Camara et al., 2002). One way to prevent this could be by chemically modifying the ring-opened AHL (e.g. by mild nucleophilic substitution or reduction of the carboxylic acid), thus preventing reconversion to the ring form. Blocking QS in the environment may have the unintentional effect of interfering with beneficial bacteria. Pseudomonas chlororaphis controls production of an antibiotic with QS. Under normal circumstances, this bacterium and its antibiotic can be used to control tomato vascular wilt caused by Fusarium oxysporum. In an experiment where the bacterium was co-cultured with an AiiA-producing bacterium, the biocontrol activity was lost, rendering the plants susceptible to infection (Molina et al., 2003).

The lactone ring is not the only chemical target point of the AHL molecules. The oxidized AHL signal molecules, such as 3-oxo-C12 HSL, can react with oxidized halogen compounds such as hypobromous and hypochlorous acids. Again, nature has developed this into a defence strategy against invading bacteria. The marine alga Laminaria digitata produces and secretes oxidized halogen compounds which interfere with QS-controlled gene expression of colonizing bacteria (Borchardt et al., 2001).

A different, enzyme-based method to inactivate the signal molecules is simply to metabolize the AHLs. Both Variovorax paradoxus and P. aeruginosa PAI-A are able to proliferate with AHLs as sole source of energy, carbon and nitrogen. The bacteria produce an amino acylase which cleaves the peptide bond of the signal molecule. The side chain is used as carbon source, the nitrogen from the amide bond is made available as ammonium via the action of lactonases and the ring part is used as energy donor (Huang et al., 2003; Leadbetter \& Greenberg, 2000).

Interestingly, differentiated human airway epithelial cells have been found to be specific with respect to breakdown of AHL molecules. The cells were able to inactivate 3-oxo-C12 HSL and C6 HSL but were unable to exert an effect on 3-oxo-C6 HSL and C4 HSL, indicating that both side chain length and oxidation state are important for this kind of inactivation (Chun et al., 2004; Hastings, 2004).

These examples demonstrate that inactivation of QS signal molecules occurs in natural environments as a functional protective strategy adopted by plants, bacteria and mammals against pathogens.

\section{Interference with the signal receptor}

A third approach to interfere with bacterial QS is to prevent the signal from being perceived by the bacteria, by either blockage or destruction of the receptor protein - the LuxR homologue.

Synthetic OSIs. One widely explored method is to block the receptor with an analogue of the AHL signal molecule. There are basically three ways to develop on the AHL scaffold: introduction of substitutions in the acyl side chain which at the same time maintain the lactone ring, introduction of substitutions and alterations in the lactone ring which at the same time leave the acyl side chain unchanged, and finally extensive modifications in both the acyl side chain and the lactone ring.

The construction of a synthetic chemical library composed of AHL derivatives differentiated in the number of carbon atoms in the side chain, including both even and uneven numbers of carbon atoms, did not result in QSI activity. In contrast, many of the compounds proved to be competitive agonists (Schaefer et al., 1996). Other groups of agonistic AHL analogues carried an acyclic or cyclic alkyl substituent on the outmost carbon atom of the side chain. However, by replacing the C-3 atom with sulphur in the acyl side chain, Persson et al. (2005) created analogues able to block expression in both LuxR- and LasR-controlled QS reporters. Other successful QSI compounds can be obtained by placing aryl substituents at the end of the side chain - at least in the 3-oxo-C6 HSL/LuxR-controlled system. If the size of the substituent is increased beyond that of a phenyl group, the antagonistic effect is lost. As the size difference of the cyclic alkyls and the aryl substituents is minimal, it is hypothesized that the aryl compounds can interact with aromatic amino acid residues in the LuxR protein, preventing normal activation (Reverchon et al., 2002). The QSI effect of the aryl AHLs can be further enhanced by replacing the C- 1 carbonyl 
group of the side chain with a sulphonyl group (Castang et al., 2004).

Probing with AHL analogue compounds carrying one or more additional atoms, including carbon, sulphur and nitrogen, in the lactone ring did not result in QSI activity (Schaefer et al., 1996). Likewise, if moieties are substituted on the C-4 atom of the ring, compounds unable to interact with the LuxR homologues are generated. Conversely, if extra moieties are placed on the C-3 ring atom, the AHL analogues are able to interact with the receptor proteins, but in this case they function as agonists (Olsen et al., 2002).

Moving further away from the cognate AHL structure, molecules retaining only part of the AHL structure have also been created and tested. A series of compounds with the $\mathrm{C} 4$ and C12 acyl side chains attached to cyclo alcohol or cyclo ketone with five or six carbon atoms resulted in a variety of agonists of the two $P$. aeruginosa QS systems. The most effective was 3-oxo-C12-(aminocyclohexanone) (Fig. 1, F), which was able to significantly lower LasR-controlled expression from a lasI- $g f p$ fusion when supplied in 100fold excess over the cognate AHL molecule (Smith et al., 2003a). Exchanging the hexanone ring for a phenolic ring resulted in the generation of a potent QSI compound (Fig. 1, G). The molar efficacy of this compound was almost 10 times higher than the previous one (Smith et al., 2003b). Interestingly, a compound with a cyclohexanol ring but otherwise similar to the two previous antagonists (Fig. 1, E) functions as an agonist of the LasR system. A similar observation was made with other compounds and QS systems. A hexane ring carrying a 3-oxo-C6 side chain showed agonistic properties, whereas antagonism was observed with a compound having the 3-oxo-C6 side chain attached to a phenyl ring (Smith et al., 2003b; Reverchon et al., 2002).

QSIs have also been identified by screening of random compound libraries. Using the above-mentioned QSIS screens, Rasmussen et al. (2005a) identified several QSI compounds with structures unrelated to the signal molecules, including 4-nitro-pyridine- $N$-oxide (4-NPO), indole, $p$-benzoquinone, 2,4,5-tribromoimidazole, indole and 3-nitrobenzene sulphone amide, the first being the most effective. 4-NPO treatment was found to block the E. coli established hybrid LuxR QS system, and further analysis demonstrated a similar effect on $P$. aeruginosa cells harbouring a $l a s B-g f p$ fusion. However, this does not necessarily mean that the compound blocks the QS regulon in $P$. aeruginosa. By the use of DNA microarray-based transcriptomics it was established that 4-NPO significantly lowers expression of $37 \%$ of the QS-regulated genes in $P$. aeruginosa, including several virulence factors known to be regulated by LasR and RhlR (Rasmussen et al., 2005a). The analysis was based on sample points taken at an optical density of $2 \cdot 0$ and only changes in gene expression greater than fivefold were taken into consideration. Taking into account that genes of the QS regulon are expressed as a continuum during the planktonic growth cycle (Hentzer et al., 2003; Schuster et al., 2003) the results strongly suggest that the LasR and RhlR receptors were targeted by the compound.

Natural QSIs. QSI compounds can be isolated from natural sources such as plants and fungi. As both plants and fungi have co-existed with QS bacteria for millions of years it can be expected that at least some of them produce QSI compounds in order to reduce the pathogenic capability of colonizing, competing and infective bacteria. In a recent screening of 50 Penicillium species grown on different growth media, a remarkably high fraction, $66 \%$, were found to produce secondary metabolites with QSI activity. Two of the compounds were identified as penicillic acid (PA) and patulin (Fig. 1), produced by Pe. radicicola and Pe. coprobium, respectively. A target validation analysis performed by DNA microarray-based transcriptomics showed that patulin targets $45 \%$ of the QS genes in $P$. aeruginosa and PA targets $60 \%$, suggesting that these two compounds indeed target the LasR and RhlR QS regulators. More circumstantial evidence for their mode of action was obtained by Western blot analysis with antibodies against the LuxR protein; this showed that the compounds accelerated turnover of LuxR (Rasmussen et al., 2005b; Koch et al., 2005). This is a similar mode of action to that of the brominated furanones from the alga Delisea pulchra (Manefield et al., 2002) (see below). Since the fungal compounds are nonbrominated this effect is not caused by the reactive bromine atoms but rather indicates that binding of blockers to the receptor induces conformational changes which lead to destabilization of the receptor. Whether this is also true for interaction with the LuxR homologues remains to be seen.

Turning to plants, crown vetch, carrot, soybean, water lily, tomato, pea seedlings (Pisum sativum), habanero (chilli) and garlic have been found to produce compounds capable of interfering with bacterial QS (Rasmussen et al., 2005a; Teplitski et al., 2000). When examined in detail, garlic extract proved to contain a minimum of three different QS inhibitors, one of which has been identified to be a cyclic disulphur compound (Rasmussen et al., 2005a; Persson et al., 2005). This QSI exerts a strong antagonistic effect on LuxR-based QS but, interestingly, has no effect against P. aeruginosa QS (Rasmussen et al., 2005a).

A much-investigated group of QSIs from a natural source is the halogenated furanone compounds produced by the macroalga D. pulchra (Givskov et al., 1996). This organism produces the molecules in central vesicle gland cells, from which they are secreted to the surface of the fronds in amounts of $100 \mathrm{ng} \mathrm{cm}^{-2}$ (Maximilien et al., 1998). Here they prevent bacterial colonization, and thereby macrofouling, by interfering with QS-controlled motility. One example of this is the ability of halogenated furanones to inhibit the QS-controlled swarming phenotype of Serratia liquefaciens MG1 (Eberl et al., 1996; Rasmussen et al., 2000). 
The QSI activity is not limited to the swr system of S. liquefaciens: the halogenated furanones also target QS systems in V. fischeri, Vibrio harveyi, Serratia ficaria and other bacteria, but the natural furanones are ineffective against $P$. aeruginosa. To circumvent this problem, chemical libraries with varying side chain length, substitution and attachment point to the furanone ring, as well as different substituents in the ring, were created. A structure-activity test revealed that synthetic derivatives devoid of an acyl side chain were highly effective against the two QS systems found in P. aeruginosa (Manefield et al., 2002; Hentzer et al., 2002, 2003; Manny et al., 1997; Hjelmgaard et al., 2003). By DNA microarray analysis it was found that expression of approximately $80 \%$ of the QS genes identified by Hentzer et al. (2003) were significantly downregulated by furanone compound 30 . The analysis was based on several sample points taken over the entire growth cycle and the analysis ignored changes in gene expression below threefold. Roughly a third of the downregulated genes have previously been reported to be major virulence factors. In good agreement with this, the furanone compound was able to downregulate production of the virulence factors protease, chitinases and pyoverdin (Hentzer et al., 2002, 2003).

\section{Global analysis of QSI effects}

So far only a few of the above-mentioned QSI compounds have been tested for their effect by means of transcriptomics or proteomics. All these investigations revealed that the QSIs identified with gene fusions do not target all or close to all QS genes found in a given bacterium (Hentzer et al., 2003; Rasmussen et al., 2005a, b; Arevalo-Ferro et al., 2003). It would be expected that a perfect QSI would show similar levels of reduction on expression of all genes constituting the QS regulon. Does this disqualify the above-mentioned compounds as QSIs? Analysis of the impact on the $P$. aeruginosa transcriptome published to date shows that generally $40-60 \%$ of the QS-regulated genes are targeted by a single QSI compound. In general, transcription of genes that are highly induced by the cognate signal molecules in a lasI rhlI knockout background is also generally significantly reduced by the QSI compounds. As already stated, the outcome of the analysis is strongly dependent on the experimental approach, in particular how many samples are taken during the cell cycle and analysed (unfortunately this number is influenced by the market price of DNA chips and chemicals/enzymes used for sample preparation). The mechanistic function of QS systems also influences the outcome. QS-regulated gene expression is a function of the internal concentration of receptor protein that is activated (i.e. acts as transcriptional activator) by signal molecules and inactivated or kept in an inactive conformation by QSIs. Previous models of the mode of action stated that the LuxR homologues became activated at a certain AHL concentration (the threshold concentration) which in turn reflects the density of the culture. However, recent research indicates that at least some of the receptor proteins in a cell are active below the threshold concentration. The 'LuxR homologue-AHL complex concentration' at which the different genes are activated merely depends on the binding affinities of the different QS-controlled promoters. Some are activated at low concentrations, early in the growth curve, whereas others need a higher concentration, being activated in later growth stages; this produces a continuum of gene expression profiles (Schuster et al., 2004; Hentzer et al., 2003). QSI compounds, on the other hand, block or destroy the LuxR homologue proteins, resulting in a lowered overall concentration; hence the genes that require higher concentrations are the ones that are more readily inhibited. The QS-controlled genes PA2591, PA2163 and PA2570 (pa1L) identified by Rasmussen et al. (2005b) support this. DNA array analysis of wild-type P. aeruginosa treated with different QSIs revealed that none of the compounds affected expression of PA2591, whereas PA2163 was downregulated by two inhibitors, 4-NPO and PA. The pa1L gene can be downregulated by $4-\mathrm{NPO}$, PA, patulin, furanone compound 30 (Fig. 1) and garlic extract (Rasmussen et al., 2005a, b; Hentzer et al., 2003). Interestingly, PA2591 is active as early as $\mathrm{OD}_{600} 0 \cdot 3$ and remains active at least up to $\mathrm{OD}_{600} 2 \cdot 7$ (Hentzer et al., 2003), indicating that activation of LasR/RhlR requires only minute amounts of AHLs for initiation of PA251 transcription. Most QSI compounds may function in a competitive manner (Hentzer et al., 2002, 2003; Rasmussen et al., 2005a, b, 2000; Manefield et al., 2002), although a recent analysis with LuxR proteins harbouring mutated amino acids in the AHL-binding cavity shows that furanone compound 30 is not a competitive inhibitor per se (Koch et al., 2005). If this extrapolates to $P$. aeruginosa there will always be at least some active AHL-receptor proteins regardless of the concentration of QSI compounds. The palL gene is only active at $\mathrm{OD}_{600} 2 \cdot 0$ or higher (Hentzer et al., 2003), indicating that it requires a high AHL/activated receptor protein concentration to be activated. This fits well with the above-mentioned finding that pa1L is inhibited by a wider range of QSIs, some of which are weaker inhibitors (i.e. they target a lower percentage of the QS-controlled genes).

\section{Inhibition of QS in vivo}

Although a QSI compound may function well in vitro it is a pretty long shot to claim that it will attenuate virulence in complex in vivo scenarios. A relatively simple model to study virulence of $P$. aeruginosa is the soil nematode Caenorhabditis elegans. These small worms normally feed on harmless bacteria, but when they graze on a lawn of $P$. aeruginosa they are rapidly killed. The factors involved in the killing have been found to include production of phenazines and $\mathrm{HCN}$, both of which are QS controlled (Mahajan-Miklos et al., 1999; Tan et al., 1999). A $100 \%$ killing of the nematodes is achieved within a few hours when the worms feed on wild-type $P$. aeruginosa. When $C$. elegans feed on $P$. aeruginosa QS knockout mutants, the killing is reduced to $10 \%$, indicating that functional QS systems are required for the expression of full virulence. The mortality of worms fed with wild-type $P$. aeruginosa in the presence of garlic extract or $4-\mathrm{NPO}$ was reduced to $40 \%$ and $5 \%$, 
respectively, indicating that these compounds could reduce virulence significantly in this in vivo model (Rasmussen et al., 2005b).

A more realistic method to evaluate the in vivo functionality of a QSI compound is by means of a pulmonary infection model. Mice are intratracheally challenged with $P$. aeruginosa embedded in alginate beads. Normally, the mucociliary escalator efficiently clears bacteria from the lungs of healthy mice. Due to the size of the alginate beads, the bacteria cannot be readily cleared and an infection which resembles the initial stages of a chronic lung infection is established. Large amounts of cells from the innate immune system, in particular neutrophile polymorphonuclear leukocytes (PMNs), are recruited to the site of infection. The destructive activities of the PMNs result in inflammation, which causes collateral damage to the tissue ( $\mathrm{Wu}$ et al., 2000). For a period of up to 14 days, this situation is reminiscent of a chronic infection in the lungs of cystic fibrosis patients (Pedersen et al., 1990). Including AHL monitor bacteria (harbouring a QS-controlled $g f p$ gene) in the alginate beads enables investigators to gain information about QS-regulated gene expression inside the lungs. If mice infected with the E. coli-based AHL monitor bacterium are injected with AHLs, green fluorescent bacteria can be seen in the lungs (Wu et al., 2000). QSI compounds can be simultaneously injected and the concentration required to block QS-dependent GFP expression in vivo can be determined (Hentzer et al., 2003). Likewise, when $P$. aeruginosa harbouring a las $B-g f p$ fusion is used to infect the mice, green fluorescent cells can be found in the lungs. If the mice are then treated with a functional QSI compound, such as furanone compound 30, the green fluorescent signal gradually disappears from the lungs of the mice within $6 \mathrm{~h}$ (Hentzer et al., 2003). As the bacteria are also tagged with a constitutively expressed $r f p$ gene their position in the tissue can be determined by expression of red fluorescence. Taken together these observations indicate that QSIs injected into the mice are able to travel to the lungs and exert their effect on infecting bacteria (Hentzer et al., 2003). The QSIs also have an effect on mortality of infected mice. Mice intratracheally challenged with $P$. aeruginosa were split into two groups. One group received treatment with saline and another with $2 \mu \mathrm{g}$ (g body weight $)^{-1}$ of furanone compound 30 . In the saline-treated group, $88 \%$ of the mice died whereas the mortality was reduced to $55 \%$ in the furanone-treated group (Hentzer et al., 2003). Similar results have been obtained with mice treated with patulin (Rasmussen et al., 2005b). The probable explanation for the lowered mortality rate observed is more rapid clearing of bacteria from the lungs in QSI-treated mice. On day 3 postinfection, mice treated with $2.5 \mu \mathrm{g}$ (g body weight) ${ }^{-1}$ of patulin had a 20 -fold lower bacterial load in the lungs compared to untreated mice (Rasmussen et al., 2005b). Likewise, mice treated with furanone compound 30 had a 1000 -fold lower number of c.f.u. per lung when treated with $0.7 \mu \mathrm{g}$ (g body weight $)^{-1}$ compared to untreated mice (Hentzer et al., 2003). A similar observation was made when mice were treated with garlic extract. Treatment with $2 \%$ $(\mathrm{v} / \mathrm{w})$ garlic extract lowered the bacterial load in the lungs by three orders of magnitude, and 5 days post-infection the mice in the treated group had cleared their lungs whereas the mice in the placebo group harboured $10^{5}$ c.f.u. per lung (Bjarnsholt et al., 2005b).

\section{Additional effects of QSIs}

Up to $80 \%$ of bacteria in nature live in organized sessile communities known as biofilms (Davies, 2003). For the clinician, biofilms pose a major problem, as they are highly tolerant to antimicrobial compounds. It has been found that bacteria living in the biofilm mode of growth are often up to 1000 -fold more tolerant to antibiotics, biocides and heavy metals than planktonic-growing cells (Allison et al., 1993; Anwar et al., 1990; Teitzel \& Parsek, 2003). Consequently, the concentrations required to eradicate bacterial biofilms often exceed the highest deliverable dose, thus precluding efficient treatment based on conventional antibacterials (Costerton et al., 1987, 1999; Drenkard, 2003; Hoiby et al., 2001). Several factors are believed to contribute to this biofilm-dependent tolerance. These include restricted penetration of antimicrobials into the biofilm; binding of the compounds to the exopolysaccharide matrix surrounding the biofilm has been mentioned as a major cause of this. The heterogeneous metabolic activity of cells located at different positions in the biofilm also adds greatly to the tolerance. At the surface of the biofilm, nutrients and oxygen are readily available and the cells grow actively. Deeper in the layers of the biofilm, where oxygen and nutrients are limited, the bacteria grow either slowly or not at all. In fact, a recent transcriptome analysis performed at different stages during biofilm development and maturation demonstrated that the bulk of biofilm cells, as judged from their gene expression profiles, must have adopted stationary-phase physiology (Hentzer et al., 2005). This explains, at least in part, why most antimicrobials which target active, growing cells are unable to fully eradicate the bacterial biofilms (Campanac et al., 2002; Drenkard, 2003; Lewis, 2001; Teitzel \& Parsek, 2003). Genes that are differently expressed in biofilms might further contribute to this tolerance. The gene pvrR (phenotypic variant regulator) was found to be involved in conversion of wild-type $P$. aeruginosa into a rough colony variant highly tolerant to antibiotics (Drenkard \& Ausubel, 2002; Drenkard, 2003). Another gene is $n d v B$. This gene confers higher tolerance to the antibiotic tobramycin at least in one P. aeruginosa isolate, PA14 (Mah et al., 2003). However, it is not differently expressed in biofilm cells of $P$. aeruginosa PAO1 (Hentzer et al., 2005) and cannot therefore be a general mechanism for tobramycin tolerance. Perhaps surprisingly, QS has also been found to be involved with the tolerance of biofilms to antimicrobial compounds. Davies et al. (1998) demonstrated that a QS-deficient lasI mutant of $P$. aeruginosa formed biofilms that were more susceptible to biocides. Likewise, biofilms formed by a las $R$ rhlR double mutant of $P$. aeruginosa is much more prone to killing by tobramycin and hydrogen peroxide than biofilms 
formed by a wild-type counterpart (Bjarnsholt et al., 2005a). These findings prompted investigators to examine whether QSI compounds would render wild-type $P$. aeruginosa biofilms susceptible to the different antimicrobials. Indeed this was the case. Hentzer et al. (2003) demonstrated that biofilms treated with furanone compounds such as furanone 30 were easily eradicated by tobramycin and were readily dispersed by detergents. Along the same lines, garlic extract, patulin and PA were found to make $P$. aeruginosa much more susceptible to killing by tobramycin (Rasmussen et al., 2005a, b). Which QS-regulated genes are involved in this increased tolerance to antimicrobials is presently unknown. The finding that biofilms formed by QS mutants or by wildtype cells treated with QSIs are more susceptible to hydrogen peroxide is intriguing, as neutrophils from the immune system utilize activated oxygen species to attack infecting bacteria. When PMNs encounter a biofilm formed by wildtype $P$. aeruginosa they are unable to kill a significant amount of the biofilm cells. In contrast, if the biofilm is formed by a QS mutant, or if a wild-type biofilm is treated with QSIs, the bacteria in the biofilm are readily killed by the PMNs (Rasmussen et al., 2005b; Bjarnsholt et al., 2005a). This observation can, at least in part, be attributed to the above-described QS-controlled tolerance. However, recent investigation indicates that the PMNs become paralysed when they encounter a wild-type $P$. aeruginosa biofilm (Bjarnsholt et al., 2005a). It was demonstrated that the PMNs were unable to develop oxidative burst and actively phagocytose the biofilm bacteria. In contrast, when the biofilm was formed by a QS mutant (or by QSI-treated wildtype bacteria) the PMNs developed an oxidative burst, performed phagocytosis and were able to eradicate the biofilm (Bjarnsholt et al., 2005a). If this is true in vivo, the synergistic action of blocking virulence and boosting the activity of the PMNs might be the reason for QSI-promoted clearing of $P$. aeruginosa from the lungs of mice.

\section{Concluding comments}

Initially the lure of interference with QS-controlled virulence poised researchers to identify different kinds of compounds or enzymes able to block QS. Certainly it was possible to develop or isolate both compounds and enzymes able to interfere with bacterial QS. At least this holds true for the relatively simple systems consisting of a QS-controlled promoter fused to a reporter. Only to a lesser extent have the identified QSIs been tested in in vivo settings. The enzymebased QSIs or quorum quenchers have successfully been applied in plant models, where the virulence of plant pathogens has been abolished. A few chemical QSIs have proven successful in the nematode and/or pulmonary mouse infection model. Unfortunately both patulin and $\mathrm{PA}$, which are able to reduce infections in mice, are mycotoxins and hence not applicable to treatment of human patients. If the garlic extract were to be used as treatment, adults would have to receive the equivalent of 50 garlic bulbs per day, an amount which probably would have severe secondary effects. However, if the active compounds from garlic can be identified and purified, they may be applicable for treatment of patients. QSI treatment (which we have denoted anti-pathogenic treatment) of pulmonary infections and in vitro biofilm experiments have demonstrated that the concentration required is similar to that found to inhibit the QS systems in planktonic cells without affecting growth or general gene expression (Hentzer et al., 2002, 2003). In addition to the first conceived strategy, inhibition of QS-controlled virulence factors, it has turned out that QSIs have effects that are more diverse. The compounds are also able to soften up bacterial biofilms, making them more susceptible to conventional antibiotics and the action of the host immune system - so researchers got more than they bargained for. On the other hand, QSIs are not magic bullets; however, combinatory chemotherapy with both antibiotics and anti-pathogenic treatment which includes a synergistic effect with the host innate immune system could form the basis of a possible future treatment scenario for chronic infections caused by bacteria which regulate pathogenicity by means of QS.

\section{Acknowledgements}

This work was supported by grants from the Danish Technical Research Council and the Villum Kann-Rasmussen Foundation to M. G.

\section{References}

Allison, C., Lai, H. C., Gygi, D. \& Hughes, C. (1993). Cell differentiation of Proteus mirabilis is initiated by glutamine, a specific chemoattractant for swarming cells. Mol Microbiol 8, 53-60.

Anwar, H., Dasgupta, M. K. \& Costerton, J. W. (1990). Testing the susceptibility of bacteria in biofilms to antibacterial agents. Antimicrob Agents Chemother 34, 2043-2046.

Arevalo-Ferro, C., Hentzer, M., Reil, G., Gorg, A., Kjelleberg, S., Givskov, M., Riedel, K. \& Eberl, L. (2003). Identification of quorumsensing regulated proteins in the opportunistic pathogen Pseudomonas aeruginosa by proteomics. Environ Microbiol 5, 1350-1369.

Bjarnsholt, T., Jensen, P. O., Burmolle, M. \& 9 other authors (2005a). Pseudomonas aeruginosa tolerance to tobramycin, hydrogen peroxide and polymorphonuclear leukocytes is quorum-sensing dependent. Microbiology 151, 373-383.

Bjarnsholt, T., Jensen, P. O., Rasmussen, T. B. \& 9 other authors (2005b). Garlic blocks quorum sensing and promotes rapid clearing of pulmonary Pseudomonas aeruginosa infections. Microbiology 151, 3873-3880.

Borchardt, S. A., Allain, E. J., Michels, J. J., Stearns, G. W., Kelly, R. F. \& McCoy, W. F. (2001). Reaction of acylated homoserine lactone bacterial signaling molecules with oxidized halogen antimicrobials. Appl Environ Microbiol 67, 3174-3179.

Byers, J. T., Lucas, C., Salmond, G. P. \& Welch, M. (2002). Nonenzymatic turnover of an Erwinia carotovora quorum-sensing signaling molecule. J Bacteriol 184, 1163-1171.

Camara, M., Williams, P. \& Hardman, A. (2002). Controlling infection by tuning in and turning down the volume of bacterial small-talk. Lancet Infect Dis 2, 667-676.

Campanac, C., Pineau, L., Payard, A., Baziard-Mouysset, G. \& Roques, C. (2002). Interactions between biocide cationic agents and bacterial biofilms. Antimicrob Agents Chemother 46, 1469-1474. 
Carlier, A., Uroz, S., Smadja, B., Fray, R., Latour, X., Dessaux, Y. \& Faure, D. (2003). The Ti plasmid of Agrobacterium tumefaciens harbors an attM-paralogous gene, aiiB, also encoding $\mathrm{N}$-acyl homoserine lactonase activity. Appl Environ Microbiol 69, 4989-4993.

Castang, S., Chantegrel, B., Deshayes, C. \& 7 other authors (2004). $\mathrm{N}$-Sulfonyl homoserine lactones as antagonists of bacterial quorum sensing. Bioorg Med Chem Lett 14, 5145-5149.

Chun, C. K., Ozer, E. A., Welsh, M. J., Zabner, J. \& Greenberg, E. P. (2004). Inactivation of a Pseudomonas aeruginosa quorum-sensing signal by human airway epithelia. Proc Natl Acad Sci U S A 101, 3587-3590.

Costerton, J. W., Cheng, K. J., Geesey, G. G., Ladd, T. I., Nickel, J. C., Dasgupta, M. \& Marrie, T. J. (1987). Bacterial biofilms in nature and disease. Annu Rev Microbiol 41, 435-464.

Costerton, J. W., Stewart, P. S. \& Greenberg, E. P. (1999). Bacterial biofilms: a common cause of persistent infections. Science 284, $1318-1322$.

Davies, D. (2003). Understanding biofilm resistance to antibacterial agents. Nat Rev Drug Discov 2, 114-122.

Davies, D. G., Parsek, M. R., Pearson, J. P., Iglewski, B. H., Costerton, J. W. \& Greenberg, E. P. (1998). The involvement of cell-to-cell signals in the development of a bacterial biofilm. Science 280, 295-298.

Diggle, S. P., Winzer, K., Chhabra, S. R., Worrall, K. E., Camara, M. \& Williams, P. (2003). The Pseudomonas aeruginosa quinolone signal molecule overcomes the cell density-dependency of the quorum sensing hierarchy, regulates rhl-dependent genes at the onset of stationary phase and can be produced in the absence of LasR. Mol Microbiol 50, 29-43.

Dong, Y. H., Xu, J. L., Li, X. Z. \& Zhang, L. H. (2000). AiiA, an enzyme that inactivates the acylhomoserine lactone quorum-sensing signal and attenuates the virulence of Erwinia carotovora. Proc Natl Acad Sci U S A 97, 3526-3531.

Dong, Y. H., Wang, L. H., Xu, J. L., Zhang, H. B., Zhang, X. F. \& Zhang, L. H. (2001). Quenching quorum-sensing-dependent bacterial infection by an $N$-acyl homoserine lactonase. Nature 411, 813-817.

Dong, Y. H., Zhang, X. F., Xu, J. L. \& Zhang, L. H. (2004). Insecticidal Bacillus thuringiensis silences Erwinia carotovora virulence by a new form of microbial antagonism, signal interference. Appl Environ Microbiol 70, 954-960.

Drenkard, E. (2003). Antimicrobial resistance of Pseudomonas aeruginosa biofilms. Microbes Infect 5, 1213-1219.

Drenkard, E. \& Ausubel, F. M. (2002). Pseudomonas biofilm formation and antibiotic resistance are linked to phenotypic variation. Nature 416, 740-743.

Eberl, L. (1999). $\mathrm{N}$-acyl homoserinelactone-mediated gene regulation in gram-negative bacteria. Syst Appl Microbiol 22, 493-506.

Eberl, L., Winson, M. K., Sternberg, C. \& 7 other authors (1996). Involvement of $\mathrm{N}$-acyl-L-hormoserine lactone autoinducers in controlling the multicellular behaviour of Serratia liquefaciens. Mol Microbiol 20, 127-136.

Engebrecht, J. \& Silverman, M. (1984). Identification of genes and gene products necessary for bacterial bioluminescence. Proc Natl Acad Sci U S A 81, 4154-4158.

Fuqua, W. C., Winans, S. C. \& Greenberg, E. P. (1994). Quorum sensing in bacteria: the LuxR-LuxI family of cell density-responsive transcriptional regulators. J Bacteriol 176, 269-275.

Gallagher, L. A., McKnight, S. L., Kuznetsova, M. S., Pesci, E. C. \& Manoil, C. (2002). Functions required for extracellular quinolone signaling by Pseudomonas aeruginosa. J Bacteriol 184, 6472-6480.

Givskov, M., de Nys, R., Manefield, M., Gram, L., Maximilien, R., Eberl, L., Molin, S., Steinberg, P. D. \& Kjelleberg, S. (1996).
Eukaryotic interference with homoserine lactone-mediated prokaryotic signalling. J Bacteriol 178, 6618-6622.

Hastings, J. W. (2004). Bacterial quorum-sensing signals are inactivated by mammalian cells. Proc Natl Acad Sci U S A 101, 3993-3994.

Hentzer, M., Riedel, K., Rasmussen, T. B. \& 9 other authors (2002). Inhibition of quorum sensing in Pseudomonas aeruginosa biofilm bacteria by a halogenated furanone compound. Microbiology 148, 87-102.

Hentzer, M., Eberl, L. \& Givskov, M. (2005). Transcriptome analysis of Pseudomonas aeruginosa biofilm development: anaerobic respiration and iron limitation. Biofilms 2, 37-61.

Hentzer, M., Wu, H., Andersen, J. B. \& 15 other authors (2003). Attenuation of Pseudomonas aeruginosa virulence by quorum sensing inhibitors. EMBO J 22, 3803-3815.

Hjelmgaard, T., Persson, T., Rasmussen, T. B., Givskov, M. \& Nielsen, J. (2003). Synthesis of furanone-based natural product analogues with quorum sensing antagonist activity. Bioorg Med Chem 11, 3261-3271.

Hoiby, N., Krogh, J. H., Moser, C., Song, Z., Ciofu, O. \& Kharazmi, A. (2001). Pseudomonas aeruginosa and the in vitro and in vivo biofilm mode of growth. Microbes Infect 3, 23-35.

Huang, J. J., Han, J. I., Zhang, L. H. \& Leadbetter, J. R. (2003). Utilization of acyl-homoserine lactone quorum signals for growth by a soil pseudomonad and Pseudomonas aeruginosa PAO1. Appl Environ Microbiol 69, 5941-5949.

Koch, B., Liljefors, T., Persson, T., Nielsen, J., Kjelleberg, S. \& Givskov, M. (2005). The LuxR receptor: the sites of interaction with quorum sensing signals and inhibitors. Microbiology 151, 3589-3602. Latifi, A., Foglino, M., Tanaka, K., Williams, P. \& Lazdunski, A. (1996). A hierarchical quorum-sensing cascade in Pseudomonas aeruginosa links the transcriptional activators LasR and RhIR (VsmR) to expression of the stationary-phase sigma factor RpoS. Mol Microbiol 21, 1137-1146.

Leadbetter, J. R. \& Greenberg, E. P. (2000). Metabolism of acylhomoserine lactone quorum-sensing signals by Variovorax paradoxus. J Bacteriol 182, 6921-6926.

Lee, S. J., Park, S. Y., Lee, J. J., Yum, D. Y., Koo, B. T. \& Lee, J. K. (2002). Genes encoding the $N$-acyl homoserine lactone-degrading enzyme are widespread in many subspecies of Bacillus thuringiensis. Appl Environ Microbiol 68, 3919-3924.

Lewis, K. (2001). Riddle of biofilm resistance. Antimicrob Agents Chemother 45, 999-1007.

Mah, T. F., Pitts, B., Pellock, B., Walker, G. C., Stewart, P. S. \& O'Toole, G. A. (2003). A genetic basis for Pseudomonas aeruginosa biofilm antibiotic resistance. Nature 426, 306-310.

Mahajan-Miklos, S., Tan, M. W., Rahme, L. G. \& Ausubel, F. M. (1999). Molecular mechanisms of bacterial virulence elucidated using a Pseudomonas aeruginosa-Caenorhabditis elegans pathogenesis model. Cell 96, 47-56.

Manefield, M. \& Turner, S. L. (2002). Quorum sensing in context: out of molecular biology and into microbial ecology. Microbiology 148, 3762-3764.

Manefield, M., Rasmussen, T. B., Henzter, M., Andersen, J. B., Steinberg, P., Kjelleberg, S. \& Givskov, M. (2002). Halogenated furanones inhibit quorum sensing through accelerated LuxR turnover. Microbiology 148, 1119-1127.

Manny, A. J., Kjelleberg, S., Kumar, N., de Nys, R., Read, R. W. \& Stainberg, P. (1997). Reinvestigation of the sulfuric acid-catalysed cyclisation of brominated 2-alkyllevulinic acids to 3-alkyl-5-methylene-2(5H)-furanones. Tetrahedron 53, 15813-15826.

Maximilien, R., de Nys, R., Holmstrom, C., Gram, L., Givskov, M., Kjelleberg, S. \& Steinberg, P. (1998). Chemical mediation of 
bacterial surface colonisation by secondary metabolites from the red alga Delisea pulchra. Aquat Microb Ecol 15, 233-246.

McKnight, S. L., Iglewski, B. H. \& Pesci, E. C. (2000). The Pseudomonas quinolone signal regulates rhl quorum sensing in Pseudomonas aeruginosa. J Bacteriol 182, 2702-2708.

Molina, L., Constantinescu, F., Michel, L., Reimmann, C., Duffy, B. \& Defago, G. (2003). Degradation of pathogen quorum-sensing molecules by soil bacteria: a preventive and curative biological control mechanism. FEMS Microbiol Ecol 45, 71-81.

Olsen, J. A., Severinsen, R., Rasmussen, T. B., Hentzer, M., Givskov, M. \& Nielsen, J. (2002). Synthesis of new 3- and 4-substituted analogues of acyl homoserine lactone quorum sensing autoinducers. Bioorg Med Chem Lett 12, 325-328.

Park, S. Y., Lee, S. J., Oh, T. K., Oh, J. W., Koo, B. T., Yum, D. Y. \& Lee, J. K. (2003). AhlD, an $N$-acylhomoserine lactonase in Arthrobacter sp., and predicted homologues in other bacteria. Microbiology 149, 1541-1550.

Parsek, M. R., Val, D. L., Hanzelka, B. L., Cronan, J. E. \& Greenberg, E. P. (1999). Acyl homoserine-lactone quorum-sensing signal generation. Proc Natl Acad Sci U S A 96, 4360-4365.

Passador, L., Tucker, K. D., Guertin, K. R., Journet, M. P., Kende, A. S. \& Iglewski, B. H. (1996). Functional analysis of the Pseudomonas aeruginosa autoinducer PAI. J Bacteriol 178, 5995-6000.

Pedersen, S. S., Shand, G. H., Hansen, B. L. \& Hansen, G. N. (1990). Induction of experimental chronic Pseudomonas aeruginosa lung infection with $P$. aeruginosa entrapped in alginate microspheres. APMIS 98, 203-211.

Persson, T., Hansen, T. H., Rasmussen, T. B., Skinderso, M. E., Givskov, M. \& Nielsen, J. (2005). Rational design and synthesis of new quorum-sensing inhibitors derived from acylated homoserine lactones and natural products from garlic. Org Biomol Chem 3, 253-262.

Pesci, E. C. \& Iglewski, B. H. (1997). The chain of command in Pseudomonas quorum sensing. Trends Microbiol 5, 132-134.

Pesci, E. C., Pearson, J. P., Seed, P. C. \& Iglewski, B. H. (1997). Regulation of las and rhl quorum sensing in Pseudomonas aeruginosa. J Bacteriol 179, 3127-3132.

Pesci, E. C., Milbank, J. B., Pearson, J. P., McKnight, S., Kende, A. S., Greenberg, E. P. \& Iglewski, B. H. (1999). Quinolone signaling in the cell-to-cell communication system of Pseudomonas aeruginosa. Proc Natl Acad Sci U S A 96, 11229-11234.

Rasmussen, T. B., Manefield, M., Andersen, J. B., Eberl, L., Anthoni, U., Christophersen, C., Steinberg, P., Kjelleberg, S. \& Givskov, M. (2000). How Delisea pulchra furanones affect quorum sensing and swarming motility in Serratia liquefaciens MG1. Microbiology 146, 3237-3244.

Rasmussen, T. B., Bjarnsholt, T., Skindersoe, M. E., Hentzer, M., Kristoffersen, P., Kote, M., Nielsen, J., Eberl, L. \& Givskov, M. (2005a). Screening for quorum-sensing inhibitors (QSI) by use of a novel genetic system, the QSI selector. J Bacteriol 187, 1799-1814.

Rasmussen, T. B., Skindersoe, M. E., Bjarnsholt, T. \& 10 other authors (2005b). Identity and effects of quorum-sensing inhibitors produced by Penicillium species. Microbiology 151, 1325-1340.

Reverchon, S., Chantegrel, B., Deshayes, C., Doutheau, A. \& CottePattat, N. (2002). New synthetic analogues of $\mathrm{N}$-acyl homoserine lactones as agonists or antagonists of transcriptional regulators involved in bacterial quorum sensing. Bioorg Med Chem Lett 12, 1153-1157.

Salmond, G. P., Bycroft, B. W., Stewart, G. S. \& Williams, P. (1995). The bacterial 'enigma': cracking the code of cell-cell communication. Mol Microbiol 16, 615-624.
Schaefer, A. L., Hanzelka, B. L., Eberhard, A. \& Greenberg, E. P. (1996). Quorum sensing in Vibrio fischeri: probing autoinducer-LuxR interactions with autoinducer analogs. J Bacteriol 178, 2897-2901.

Schuster, M., Lostroh, C. P., Ogi, T. \& Greenberg, E. P. (2003). Identification, timing, and signal specificity of Pseudomonas aeruginosa quorum-controlled genes: a transcriptome analysis. J Bacteriol 185, 2066-2079.

Schuster, M., Urbanowski, M. L. \& Greenberg, E. P. (2004). Promoter specificity in Pseudomonas aeruginosa quorum sensing revealed by DNA binding of purified LasR. Proc Natl Acad Sci U S A 101, 15833-15839.

Seed, P. C., Passador, L. \& Iglewski, B. H. (1995). Activation of the Pseudomonas aeruginosa lasI gene by LasR and the Pseudomonas autoinducer PAI: an autoinduction regulatory hierarchy. J Bacteriol 177, 654-659.

Smith, K. M., Bu, Y. \& Suga, H. (2003a). Induction and inhibition of Pseudomonas aeruginosa quorum sensing by synthetic autoinducer analogs. Chem Biol 10, 81-89.

Smith, K. M., Bu, Y. \& Suga, H. (2003b). Library screening for synthetic agonists and antagonists of a Pseudomonas aeruginosa autoinducer. Chem Biol 10, 563-571.

Tan, M. W., Mahajan-Miklos, S. \& Ausubel, F. M. (1999). Killing of Caenorhabditis elegans by Pseudomonas aeruginosa used to model mammalian bacterial pathogenesis. Proc Natl Acad Sci U S A 96, 715-720.

Teitzel, G. M. \& Parsek, M. R. (2003). Heavy metal resistance of biofilm and planktonic Pseudomonas aeruginosa. Appl Environ Microbiol 69, 2313-2320.

Teplitski, M., Robinson, J. B. \& Bauer, W. D. (2000). Plants secrete substances that mimic bacterial $\mathrm{N}$-acyl homoserine lactone signal activities and affect population density-dependent behaviors in associated bacteria. Mol Plant Microbe Interact 13, 637-648.

Uroz, S., D’Angelo-Picard, C., Carlier, A., Elasri, M., Sicot, C., Petit, A., Oger, P., Faure, D. \& Dessaux, Y. (2003). Novel bacteria degrading $\mathrm{N}$-acylhomoserine lactones and their use as quenchers of quorum-sensing-regulated functions of plant-pathogenic bacteria. Microbiology 149, 1981-1989.

Van Delden, C. \& Iglewski, B. H. (1998). Cell-to-cell signaling and Pseudomonas aeruginosa infections. Emerg Infect Dis 4, 551-560.

Wagner, V. E., Bushnell, D., Passador, L., Brooks, A. I. \& Iglewski, B. H. (2003). Microarray analysis of Pseudomonas aeruginosa quorum-sensing regulons: effects of growth phase and environment. J Bacteriol 185, 2080-2095.

Wang, L. H., Weng, L. X., Dong, Y. H. \& Zhang, L. H. (2004). Specificity and enzyme kinetics of the quorum-quenching $\mathrm{N}$-acyl homoserine lactone lactonase (AHL-lactonase). J Biol Chem 279, 13645-13651.

Winson, M. K., Camara, M., Latifi, A. \& 7 other authors (1995). Multiple $N$-acyl-L-homoserine lactone signal molecules regulate production of virulence determinants and secondary metabolites in Pseudomonas aeruginosa. Proc Natl Acad Sci U S A 92, 9427-9431.

Wu, H., Song, Z., Hentzer, M. \& 8 other authors (2000). Detection of $\mathrm{N}$-acylhomoserine lactones in lung tissues of mice infected with Pseudomonas aeruginosa. Microbiology 146, 2481-2493.

Yates, E. A., Philipp, B., Buckley, C. \& 8 other authors (2002). NAcylhomoserine lactones undergo lactonolysis in a $\mathrm{pH}$-, temperature-, and acyl chain length-dependent manner during growth of Yersinia pseudotuberculosis and Pseudomonas aeruginosa. Infect Immun 70, 5635-5646. 